\title{
miR-33a-5p inhibits the growth and metastasis of melanoma cells by targeting SNAI2
}

\author{
Z. R. ZHANG, N. YANG* \\ Department of Plastic Surgery, The Affiliated Hospital of Beihua University, Jilin City, Jilin Province, China \\ ${ }^{*}$ Correspondence: imdswq@163.com
}

Received August 23, 2019 / Accepted October 23, 2019

\begin{abstract}
MicroRNAs have been verified as critical regulators in the development of melanoma. miR-33a-5p was significantly downregulated in melanoma, however, the specific role and regulatory mechanism of miR-33a-5p in melanoma were still unclear. The present study identified that miR-33a-5p was downregulated in melanoma tissues and cells, while SNAI2 was upregulated. miR-33a-5p directly targeted SNAI2 and negatively regulated its expression in melanoma cells. Overexpression of miR-33a-5p repressed proliferation, migration, invasion, EMT and promoted apoptosis of melanoma cell in vitro, these effects were partially reversed by SNAI2 overexpression. In addition, miR-33a-5p impaired melanoma growth in vivo by inhibiting SNAI2. Mechanistically, miR-33a-5p repressed activation of the PI3K/AKT/mTOR pathway by targeting SNAI2. In conclusion, miR-33a-5p repressed the progression of melanoma by targeting SNAI2 via inactivation of the PI3K/AKT/ mTOR signaling pathway, providing a potential molecular mechanism for the treatment of melanoma.
\end{abstract}

Key words: melanoma, miR-33a-5p, SNAI2, PI3K/AKT/mTOR

Malignant melanoma is an aggressive skin malignancy that originates from the transformation of melanocytes and leads to many skin cancer-related deaths [1]. And it ranks fifth in men and seventh in women in all malignant tumors [2]. The incidence of melanoma is increasing every year in the world [3]. Although treatments for melanoma were improved, advanced melanoma could not be well treated [4]. Therefore, it is necessary to find novel markers for the diagnosis of melanoma.

MicroRNAs (miRNAs) are small non-coding RNAs with 18-22 nucleotides [5]. miRNAs regulate the target genes expression by complementing the 3' UTR region of mRNAs [6]. Numerous studies indicated that miRNAs could act as pivotal regulatory factors in various biological processes, including cell growth and metastasis $[7,8]$. Therefore, changes in miRNA expression were associated with tumor progression. A growing number of studies indicated that miRNAs were maladjusted in a variety of cancers including melanoma. For example, Yang et al. claimed that miR-150-5p suppressed cell growth, migration, and invasion by modulating SIX1 expression in melanoma [9]. Sun et al. declared that miR-431 impaired cell progression by targeting NOTCH2 in melanoma [10]. Ma et al. also demonstrated that miR-23a-3p inhibited mucosal melanoma progress via targeting adenylate cyclase 1 and impairing cAMP and
MAPK pathways [11]. Therefore, it is of great significance to study the mechanism of miRNAs in melanoma for the diagnosis and treatment of this disease.

Previous studies identified that miR-33a-5p served as an anti-tumor factor in most cancers, including colorectal cancer [12], glioma [13] and tongue squamous carcinoma [14]. Although Cao et al. found that miR-33a-5p was drastically downregulated and enhanced the radiosensitivity by repressing glycolysis in melanoma [15], the idiographic mechanism of miR-33a-5p action in melanoma is still unclear.

The SNAIL family is the most widely studied epithelial-mesenchymal transition (EMT) associated transcription factor [16]. Snail Family Transcriptional Repressor 2 (SNAI2), also known as a slug, was found to be critical in chick embryo mesoderm formation and neural crest emigration [17]. More recently, it has been reported that SNAI2 was significantly aggrandized in various cancers $[18,19]$. Also, it was shown that SNAI2 functioned as a melanocyte-specific factor; which was required for the metastases of melanoma [20]. However, the exact role of SNAI2 in melanoma remains unclear. Some studies have demonstrated that the PI3K/ AKT/mTOR signaling pathway could regulate the growth and metastasis of melanoma cells [21,22]. However, whether miR-33a-5p could regulate the PI3K/AKT/mTOR signaling pathway in melanoma has not been investigated. 
To date, the molecular mechanism of miR-33a-5p in regulating SNAI2 in melanoma has not been studied. The study was mainly to explore the impact of miR-33a-5p/ SNAI2 axis on the development of melanoma, providing a new clue for the treatment of melanoma.

\section{Patients and methods}

Clinical specimens and cell lines. Melanoma tissues and normal tissues were acquired from 29 patients at the Affiliated Hospital of Beihua University. All patients signed informed consent, and the experiments were approved by the Ethics Committee of the Affiliated Hospital of Beihua University. Tissues were separated and kept in liquid nitrogen.

Human melanoma cell lines (SKMEL-28, A375, WM35, and SKMEL-1) and melanocyte cell line PIG1 were purchased from TongPai (Shanghai, China). They were grown in RPMI 1640, including 10\% FBS (Thermo Fisher Scientific, Waltham, MA, USA), $1 \%$ penicillin/streptomycin at $37^{\circ} \mathrm{C}$ with $5 \% \mathrm{CO}_{2}$.

Transfection assay. miR-33a-5p mimic, miR-33a-5p inhibitor and their NCs (mimic-NC and inhibitor-NC) were synthesized by GenePharma (Shanghai, China). SNAI2 overexpression vectors and control pcDNA were bought from SyngenTech (Beijing, China). Cells that had not undergone any treatment were used as controls. For transfection, Lipofectamine 2000 (Invitrogen, Carlsbad, CA, USA) was used.

Reverse transcription-quantitative polymerase chain reaction (RT-qPCR). RNA from melanoma tissues and cells was isolated by Trizol (Thermo Fisher Scientific). RNA was reverse-transcribed into cDNA by a PrimerScript RT kit (TaKaRa Bio Technology, Wuhan, China). RT-qPCR was

Table 1. The relationship between miR-33a-5p expression level and the clinicopathological factors in melanoma tissues.

\begin{tabular}{|c|c|c|c|c|}
\hline \multirow{2}{*}{ Parameter } & \multirow{2}{*}{ Case } & \multicolumn{2}{|c|}{ miR-33a-5p expression } & \multirow{2}{*}{ p-value ${ }^{a}$} \\
\hline & & High $(n=29)$ & Low $(n=29)$ & \\
\hline \multicolumn{5}{|l|}{ Gender } \\
\hline Male & 25 & 15 & 10 & 0.124 \\
\hline Female & 32 & 15 & 17 & \\
\hline \multicolumn{5}{|l|}{ Age (years) } \\
\hline$\leq 54$ & 25 & 14 & 9 & 0.314 \\
\hline$>54$ & 38 & 16 & 22 & \\
\hline \multicolumn{5}{|c|}{ Tumor size (mm) } \\
\hline$\leq 1 \mathrm{~cm}$ & 25 & 16 & 9 & $0.024^{*}$ \\
\hline$>1 \mathrm{~cm}$ & 33 & 14 & 19 & \\
\hline \multicolumn{5}{|l|}{ STM stage } \\
\hline I-II & 26 & 17 & 8 & $0.005^{*}$ \\
\hline III & 30 & 9 & 22 & \\
\hline \multicolumn{5}{|c|}{ Lymph node metastasis } \\
\hline No & 33 & 19 & 14 & $0.024^{*}$ \\
\hline Yes & 24 & 14 & 10 & \\
\hline
\end{tabular}

${ }^{\star}$ Indicated statistical significance $(\mathrm{p}<0.05)$ conducted on Real-Time PCR System (Applied Biosystems, Carlsbad, CA, USA). SNAI2 expression was standardized to glyceraldehyde-3-phosphate dehydrogenase (GAPDH) and the relative expression of $\mathrm{miR}-33 \mathrm{a}-5 \mathrm{p}$ was standardized to U6. The $2^{-\Delta \Delta \mathrm{Ct}}$ method was applied to calculate the data. Sequence of primers: miR-33a-5p Forward: 5'-GATCCTCAGTGCATTGTAGTTGC-3', Reverse: 5'-CTCTGTCTCTCGTCTTGTTGGTAT-3'. U6 Forward: 5'-ATTGGAACGATACAGAGAAGATT-3'; Reverse: 5'-GGAACGCTTCACGAATTTG-3'. SNAI2 Forward: 5'-CTGGGCTGGCCAAACATAAG-3'; Reverse: 5'-CCTTGTCACAGTATTTACAGCTGAAAG-3'. GAPDH Forward: 5'-GGCTGTTGTCATACTTCTCATGG-3'; Reverse: 5'-AGGAAAAGCATCACCCGGAG-3'.

Cell proliferation and apoptosis assays. For cell proliferation of SKMEL-28 and WM35, cells were grown in plates at the density of 2000 cells/well. At $0 \mathrm{~h}, 24 \mathrm{~h}, 48 \mathrm{~h}$, and $72 \mathrm{~h}$ post-transfection, $10 \mu \mathrm{l}(5 \mathrm{mg} / \mathrm{ml})$ of methylthiazolyldiphenyl-tetrazolium bromide (MTT) (Gefanbio, Wuhan, China) were added to each well and cells were cultivated for $3 \mathrm{~h}$. Then, $150 \mu \mathrm{l}$ of dimethyl sulfoxide (DMSO) was used to solubilize the formazan salt. Cells that had not undergone any treatment were used as controls. Finally, the absorbance at $490 \mathrm{~nm}$ was measured using a microplate reader.

For apoptotic rate detection, $5 \times 10^{4}$ of SKMEL-28 and WM 35 cells were inoculated in plates. The plasmid was transfected. $48 \mathrm{~h}$ later, cells were washed and dyed with Annexin V-FITC and propidium iodide (PI) (BD Biosciences, Franklin Lake, NJ, USA), followed by flow cytometry. Cells that had not undergone any treatment were used as controls.

Western blot assay. Total protein from melanoma tissues and cells was collected, lysed by using the RIPA buffer (Beyotime, Jiangsu, China) and measured by a BCA kit (Thermo Fisher Scientific). Protein was then separated and transferred to a polyvinylidene fluoride (PVDF) membrane (Thermo Fisher Scientific). The bands were incubated with skim milk powder and incubated by primary antibodies at $4^{\circ} \mathrm{C}$ for $12 \mathrm{~h}$. Next, the bands were probed with the secondary antibodies and visualized via the ECL reagent (Amersham Biosciences, Buckinghamshire, UK). Antibodies were as follows: Bcl-2 (1:50, MA5-11757, Invitrogen), Bax (1:2000, ab53154, Abcam, Cambridge, UK), cleaved-caspase3 (1:1000, ab32042, Abcam), N-cadherin (1:100, ab18203, Abcam), E-cadherin (1:50, ab1416, Abcam), Vimentin (1:1000, ab137321, Abcam), Snail (1:1000, ab53519, Abcam), SNAI2 (1:500, ab180714, Abcam), PI3K (1:1000, ab1678, Abcam), p-AKT308 (1:1000, \#2965, Santa Cruz Biotechnology, Shanghai, China), AKT (1:3000, ab179463, Abcam), mTOR (1:2000, ab2732, Abcam), GAPDH (1:3000, MA5-15738-HRP, Thermo Fisher Scientific) and HRP-labeled secondary antibody (1:5000, ab52614, Abcam).

Transwell assay. SKMEL-28 and WM35 cells were starved for $12 \mathrm{~h}$ before inoculation and were then sowed into the upper chamber of 24-well plates containing an uncoated or Matrigel-coated insert (BD Biosciences) with $100 \mu \mathrm{l}$ of serum-free medium to detect cell migration and invasion, 
respectively. $600 \mu \mathrm{l}$ of DMEM containing fetal bovine serum was added to the lower chambers. After one day, the cells on the lower surface of the upper chamber were fixed in $4 \%$ polyoxymethylene and stained with $0.1 \%$ crystal violet. Cells that had not undergone any treatment were used as controls. The migratory or invading SKMEL-28 and WM35 cells were observed under a microscope.

Dual-luciferase reporter assay. First, the binding sites of the SNAI2 3'UTR region and miR-33a-5p were predicted by TargetScan. The wild type (WT-3'UTR) and mutant type (MUT-3'UTR) plasmids of SNAI2 containing miR-33a-5p binding sites or not were transfected into cells using Lipofectamine 2000 (Invitrogen). Two days later, the luciferase activity was assessed using a dual-luciferase reporter system (Promega, Madison, WI, USA).

Tumor xenograft assay. SKMEL-28 cells $\left(8 \times 10^{6}\right)$ transfected with miR-33a-5p mimic or mimic-NC were subcutaneously injected into the five-week old nude mice. One week later, the tumor volume was estimated every week for 4 weeks. After $28 \mathrm{~d}$, the mice were euthanized, and the formed tumors were resected and weighed. The resected tumors were frozen in liquid nitrogen. The protocol of animal experiments was approved by the Animal Care Committee of the Affiliated Hospital of Beihua University.

Statistical analysis. All results were presented as mean \pm standard deviation (SD) and conducted at least three selfgoverned replicates. Pearson's coefficient assay was applied for expression correlation. Student's t-test was used to estimate obvious differences. ${ }^{\star} \mathrm{p}<0.05$ indicated a statistically significant difference.

\section{Results}

miR-33a-5p was downregulated in melanoma tissues and cell lines. To explore the expression of miR-33a-5p in melanoma, qRT-PCR was performed. The results showed that the expression of miR-33a-5p was remarkably downregulated in melanoma tissues compared with normal tissues (Figure 1A). Further analysis of the relation- ship between clinicopathological features of melanoma and miR-33a-5p expression demonstrated that the low expression of miR-33a-5p in melanoma tissues contributed to the lymph-node metastasis, tumor size, and high STM stage (Table 1). Next, the expression of miR-33a-5p was measured in human normal melanocytes and melanoma cells. The results indicated that miR-33a-5p was obviously downregulated in SKMEL-28, A375, WM35, and SKMEL-1 melanoma cell lines compared with the PIG1 cell line (Figure 1B). The results demonstrated that the expression of miR-33a-5p was decreased in melanoma tissues and cell lines.

miR-33a-5p repressed proliferation and promoted apoptosis of melanoma cells in vitro. To investigate the functional role of miR-33a-5p in melanoma cells, gainof-function experiments were performed by transfecting miR-33a-5p mimic into SKMEL-28 and WM35 cells. miR-33a-5p expression was significantly upregulated in SKMEL-28 and WM35 cells transfected with miR-33a-5p mimic (Figures 2A, 2B). MTT assay revealed that the proliferation of SKMEL-28 and WM35 cells transfected with miR-33a-5p mimic was significantly inhibited compared with the control (Figures 2C, 2D). Flow cytometry assay showed that the apoptotic rates of SKMEL-28 and WM35 cells transfected with miR-33a-5p mimic were significantly increased compared with NCs (Figure 2E). Meanwhile, the results of western blot showed a notably decreased Bcl-2 expression and a markedly increased Bax and cleavedcaspase 3 expression in SKMEL-28 and WM35 cells transfected with miR-33a-5p mimic, while there was no obvious change in caspase 3 expression (Figures 2F, 2G). All data supported that miR-33a-5p effectively inhibited proliferation but promoted apoptosis of melanoma cells, indicating that miR-33a-5p impaired the growth of melanoma cells.

miR-33a-5p impeded migration, invasion, and EMT of melanoma cells in vitro. In order to explore the metastatic effect of miR-33a-5p on melanoma cells, transwell assay and western blot were used. The results showed that the migration and invasion of SKMEL-28 and WM35 cells were exceptionally constrained after overexpression of miR-33a-5p
A

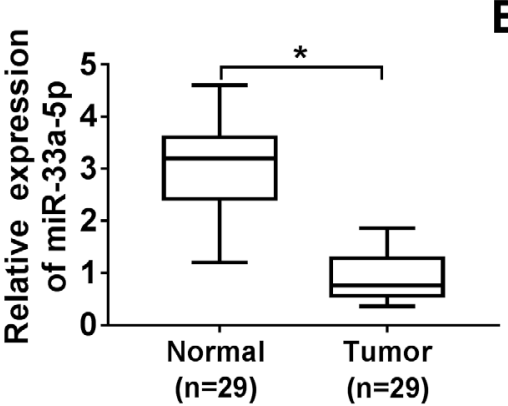

B

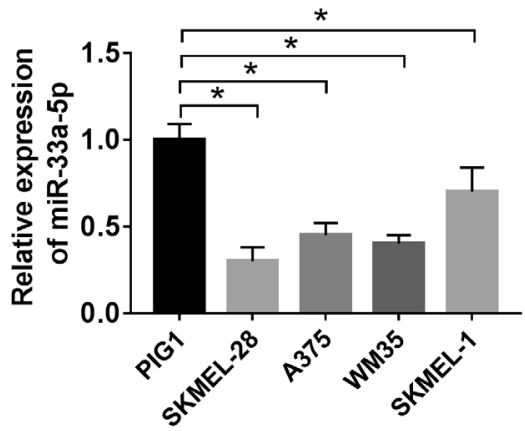

Figure 1. miR-33a-5p was downregulated in melanoma tissues and cell lines. A) The expression of miR-33a-5p in melanoma tissues was measured by qRT-PCR in comparison with corresponding normal tissues $(n=29)$. B) miR-33a-5p expression in human melanocyte cell line PIG1 and melanoma cell lines SKMEL-28, A375, WM35, and SKMEL-1 was detected by qRT-PCR. * ${ }^{*}<0.05$ 
A

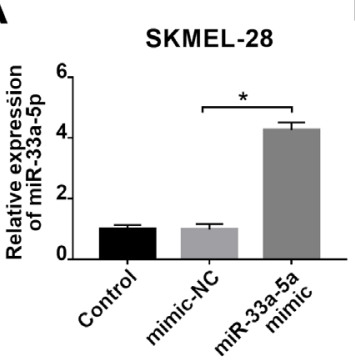

E

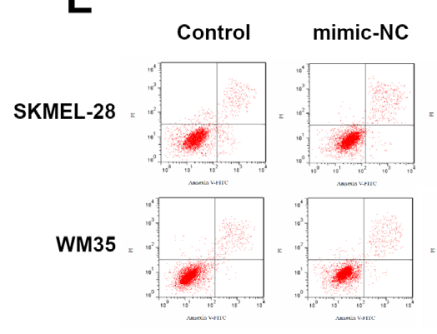

G

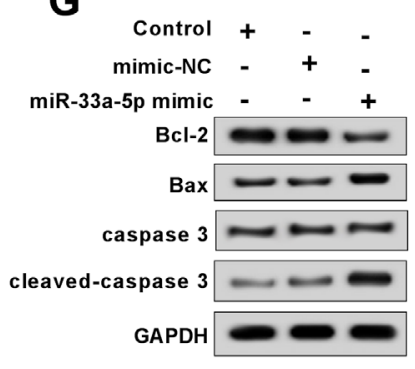

B

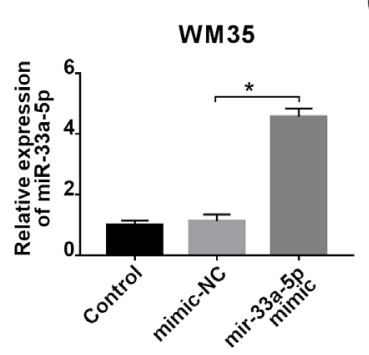

C

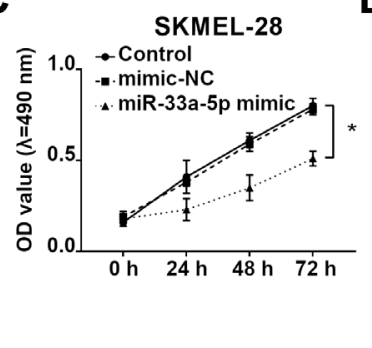

D

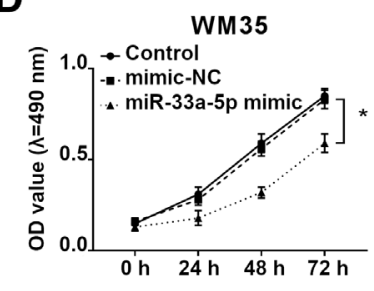

$\mathbf{F}$

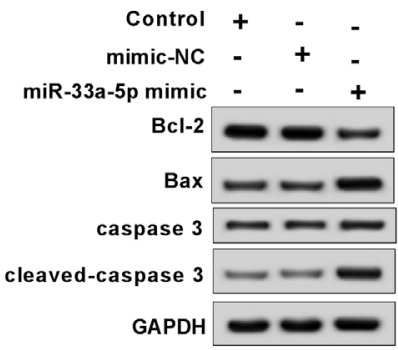

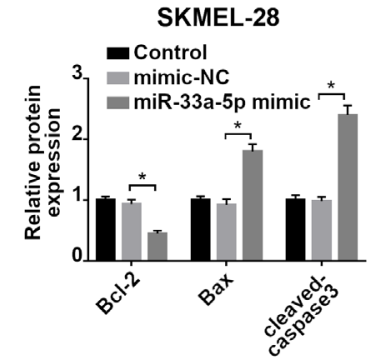

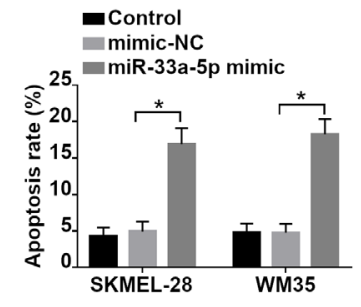

WM35

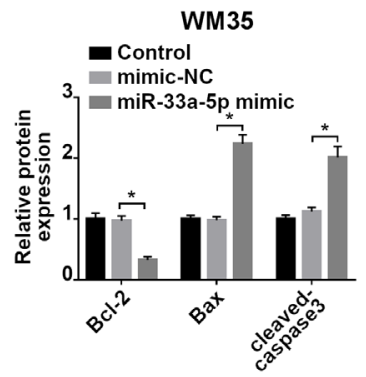

Figure 2. miR-33a-5p repressed proliferation and promoted apoptosis of melanoma cells in vitro. A, B) miR-33a-5p expression was determined by qRT-PCR in SKMEL-28 and WM35 cells transfected with control, mimic NC or miR-33a-5p mimic. C, D) The proliferation of SKMEL-28 and WM35 cells transfected with control, mimic NC or miR-33a-5p mimic was measured by MTT assay. E) The apoptotic rate of SKMEL-28 and WM35 cells was measured by flow cytometry. F, G) The protein expression levels of Bcl-2, Bax, caspase 3, and cleaved-caspase 3 in SKMEL-28 and WM35 cells were detected by western blot. ${ }^{*} \mathrm{p}<0.05$

(Figures 3A, 3B). In addition, overexpression of miR-33a-5p in SKMEL-28 and WM35 cells markedly decreased the expression levels of EMT-related protein Vimentin, $\mathrm{N}$-cadherin and Snail, while the expression of E-cadherin was notably increased (Figures 3C, 3D). The above data indicated that miR-33a-5p could repress migration, invasion, and EMT of melanoma cells.

SNAI2 was a direct target of miR-33a-5p. To identify the potential targets of miR-33a-5p that might contribute to its tumor growth-inhibitory effects, we predicted its target genes through bioinformatics. It was predicted by TargetScan that there were binding sites between miR-33a-5p and SNAI2 3'UTR (Figure 4A). Besides, the dual-luciferase reporter assay showed that the luciferase activity in SKMEL-28 and WM35 cells transfected with SNAI2-3' UTR-WT and miR-33a-5p mimic was strikingly constrained compared to that in cells transfected with SNAI2-3' UTR-WT and miR-NC, while there was no obvious change in the cells transfected with SNAI2-3' UTR-MUT (Figures 4B, 4C).
Moreover, we analyzed the mRNA and protein levels of SNAI2 in SKMEL-28 and WM35 cells transfected with miR-33a-5p mimic or inhibitor. We found that interference with miR-33a-5p significantly augmented the SNAI2 expression in SKMEL-28 and WM35 cells, at both the mRNA and protein levels, and vice versa (Figures 4D, 4G). Furthermore, the RT-qPCR analysis indicated that the expression of SNAI2 in melanoma tissues was drastically increased and negatively correlated with the expression of miR-33a-5p (Figures $4 \mathrm{H}$, 4I). Consistently, the mRNA and protein expression levels of SNAI2 were obviously upregulated in melanoma cell lines compared with normal cells (Figures 4J, 4K). These data supported that miR-33a-5p directly targeted SNAI2.

miR-33a-5p inhibited proliferation and promoted apoptosis of melanoma cells by regulating SNAI2. To determine whether SNAI2 was involved in miR-33a-5pmediated regulation on melanoma progression, we restored SNAI2 expression in SKMEL-28 and WM35 cells transfected with miR-33a-5p mimic (miR-33a-5p). First, the 

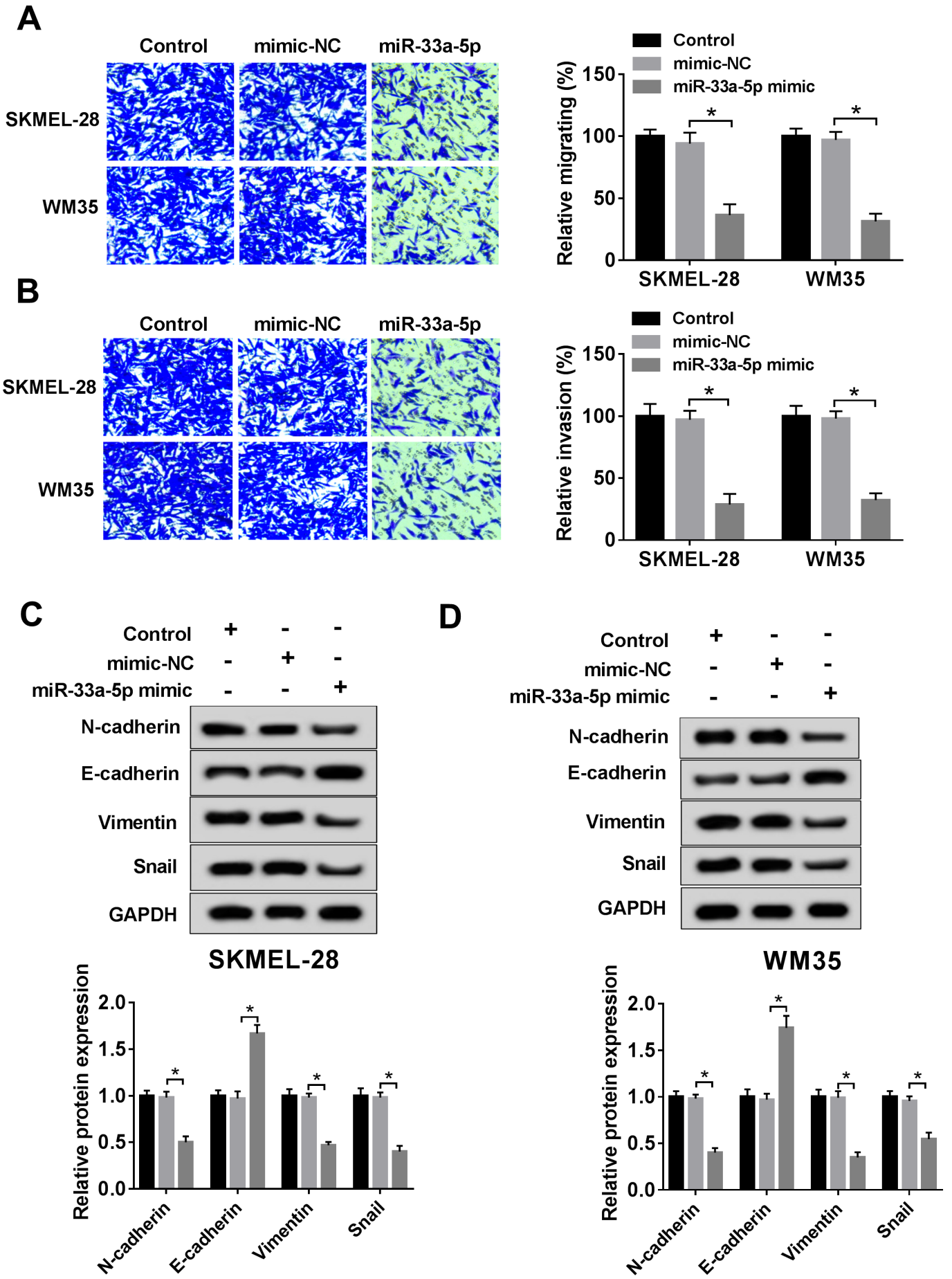

Figure 3. miR-33a-5p hindered migration, invasion, and EMT of melanoma cells in vitro. A, B) Transwell assay was applied to detect migration and invasion of SKMEL-28 and WM35 cells transfected with control, mimic NC or miR-33a-5p mimic. C, D) Western blot assay was conducted to measure the protein expression levels of N-cadherin, E-cadherin, Vimentin, and Snail in SKMEL-28 and WM35 cells. ${ }^{*} \mathbf{p}<0.05$

mRNA and protein expression levels of SNAI2 were significantly repressed in SKMEL-28 and WM35 cells transfected with miR-33a-5p, and overexpression of SNAI2 inverted this inhibition (Figures 5A-C). As expected, MTT assay showed that the overexpression of SNAI2 in SKMEL-28 and WM35 cells enhanced the effects on cell proliferation (Figures 5D, 5E). Besides, the apoptotic rate was notably impaired in SKMEL-28 and WM35 cells transfected with 


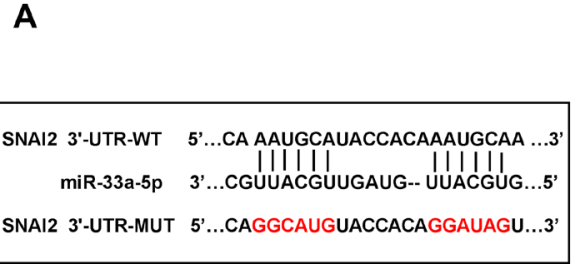

E

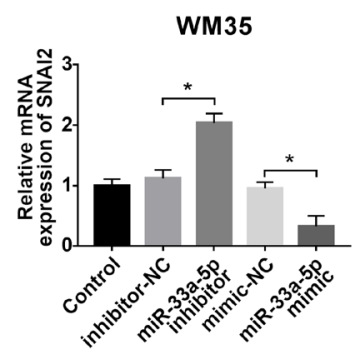

I

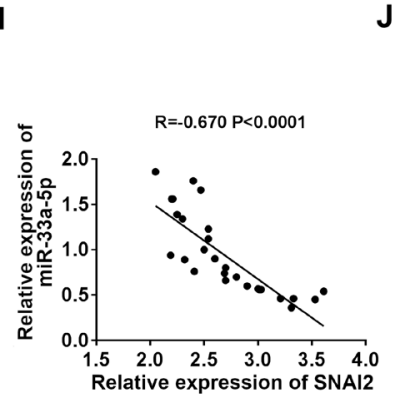

B

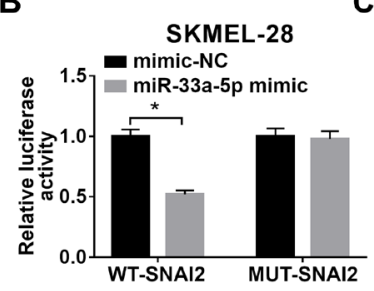

C

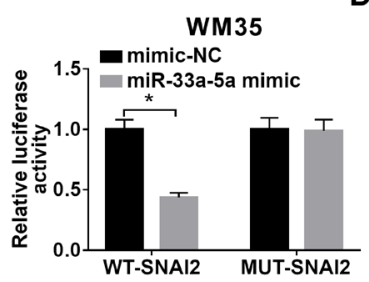

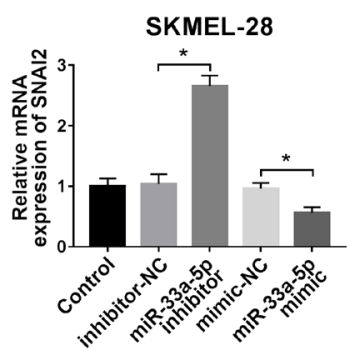

F
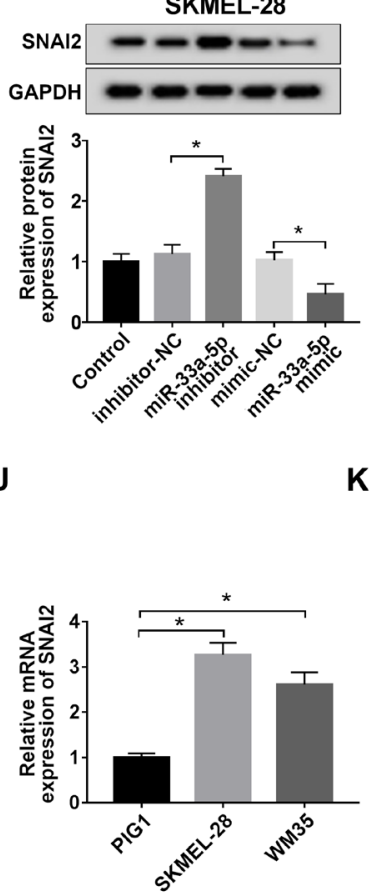

K
G
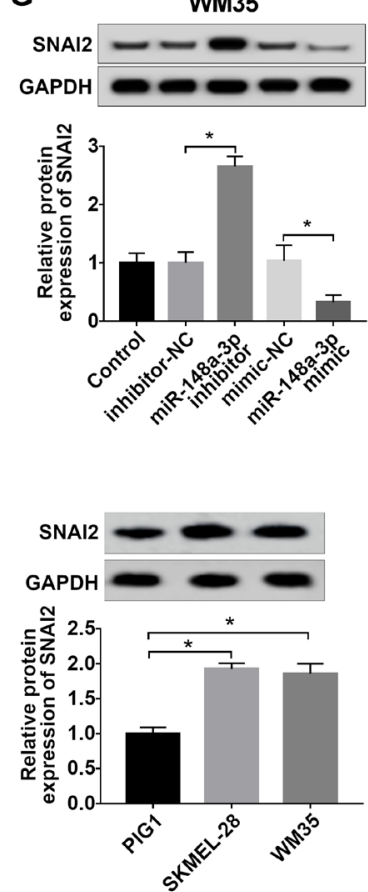

H

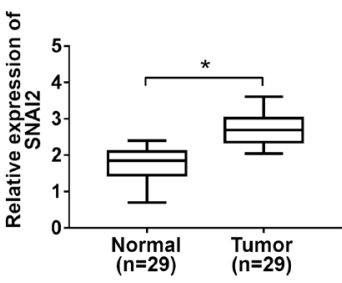

Figure 4. Identification of miR-33a-5p targeting SNAI2. A) The binding sites of miR-33a-5p in the 3'UTR region of SNAI2 were predicted by TargetScan. B, C) The luciferase activity of SKMEL-28 and WM35 cells co-transfected SNAI2-3' UTR-WT or SNAI2-3' UTR-MUT with miR-33a-5p or mimic NC was checked by dual-luciferase reporter assay. D, E) The mRNA expression of SNAI2 in SKMEL-28 and WM35 cells transfected with miR33a-5p inhibitor, miR-33a-5p mimic, control or their NCs was measured by qRT-PCR. F, G) The protein expression of SNAI2 in SKMEL-28 and WM35 cells was measured by western blot. $\mathrm{H}$ ) The expression of SNAI2 in melanoma tissues was measured by qRT-PCR compared with normal tissues $(\mathrm{n}=29)$. I) Correlation between the expression levels of miR-33a-5p and SNAI 2 was analyzed by Spearman's test $\left(R^{2}=0.670, p<0.0001\right)$. J, K) The mRNA and protein expression levels of SNAI2 were detected by $q$ RT-PCR in melanoma cell lines SKMEL-28 and WM 35 and melanocyte cell line PIG1. ${ }^{*} \mathrm{p}<0.05$

SNAI2 and miR-33a-5p compared with cells transfected with pcDNA-NC and miR-33a-5p (Figure 5F). Additionally, the increased expression levels of Bax and cleaved-caspase 3 mediated by miR-33a-5p and the decreased expression of $\mathrm{Bcl}-2$ induced by miR-33a-5p were able to recover after overexpression of SNAI2 in SKMEL-28 and WM35 cells (Figures 5G, 5H). These results indicated that miR-33a-5p suppressed the growth of melanoma cells by targeting SNAI2.

Overexpression of SNAI2 rescued the migration, invasion, and EMT impeded by miR-33a-5p. To investigate whether miR-33a-5p directly regulates the metastasis of melanoma cells by targeting SNAI2, transwell and western blot assays were used. The results showed that miR-33a-5p could inhibit the migration and invasion of SKMEL-28 and WM35 cells, whereas the inhibitory effects of miR-33a-5p could be restored by the overexpression of SNAI2 (Figures 6A, 6B). In addition, the overexpression of miR-33a-5p in SKMEL-28 and WM35 cells markedly decreased the protein levels of Vimentin, N-cadherin, Snail and increased the E-cadherin expression, while this inhibition or promotion could be regained after the overexpression of SNAI2 (Figures 6C, 6D). Taken together, our results demonstrated that miR-33a-5p suppressed the migration, invasion, and EMT of melanoma cells by targeting SNAI2.

miR-33a-5p inhibited tumor growth in vivo. To evaluate the impact of miR-33a-5p on tumor growth in vivo, we performed a xenograft experiment. Consistent with the results in vitro, we found that the overexpression of miR-33a-5p retarded the tumor volume and weight (Figures 7A, 7B). Moreover, we also analyzed the changes 
A

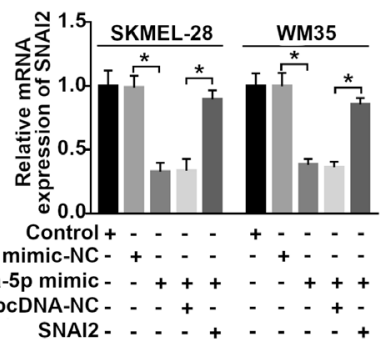

D

SKMEL-28

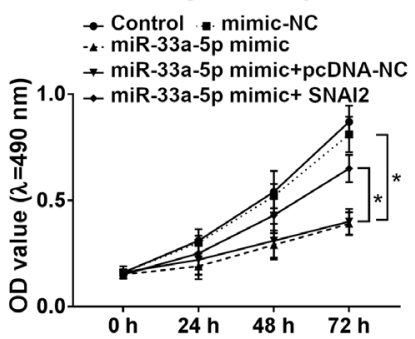

B
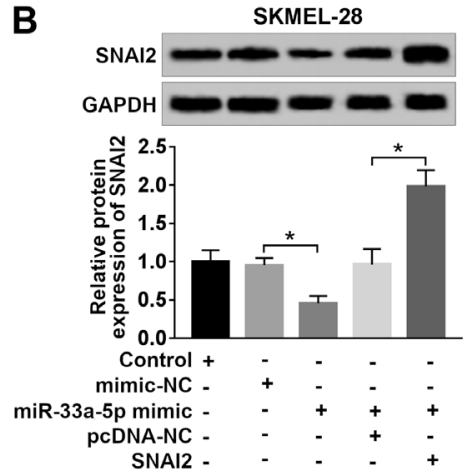

E

WM35

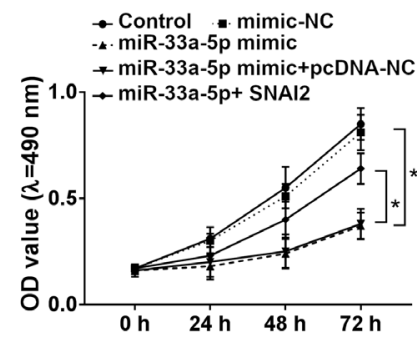

C
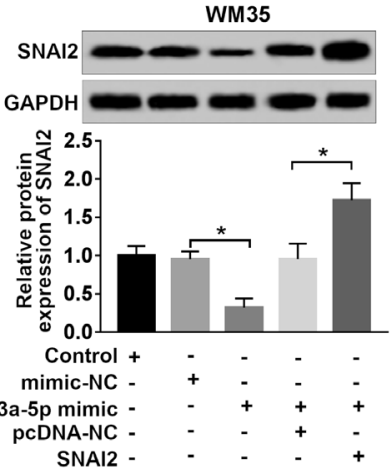

F

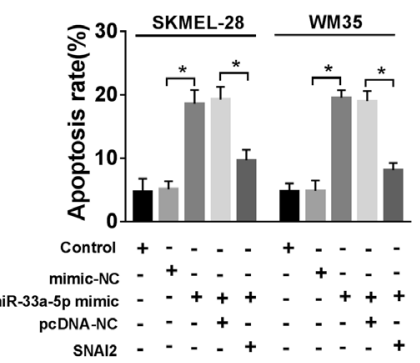

G

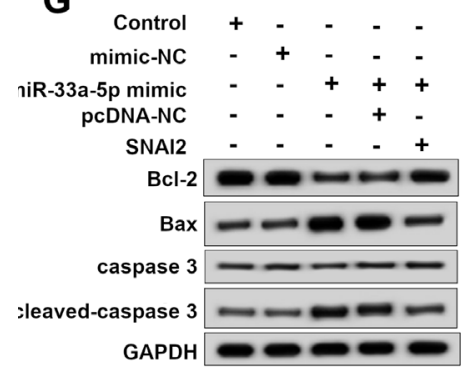

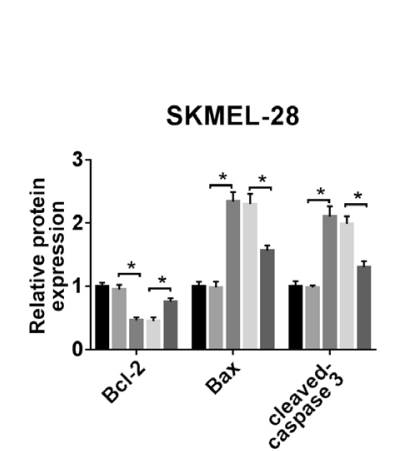

$\mathrm{H}$

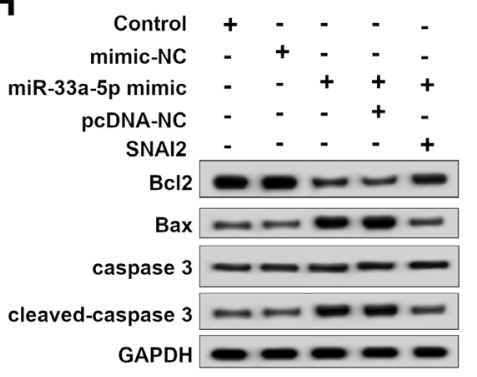

WM35

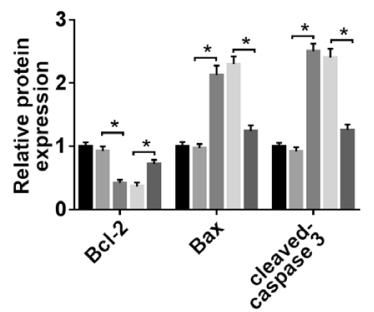

Figure 5. miR-33a-5p regulated the proliferation and apoptosis of melanoma cells by regulating SNAI2. A-C) The mRNA and protein expression levels of SNAI2 were detected in SKMEL-28 and WM35 cells transfected with control, mimic NC, miR-33a-5p mimic + pcDNA-NC or miR-33a-5p mimic + SNAI 2 by $q$ RT-PCR and western blot assays. D, E) The proliferation of SKMEL-28 and WM35 cells was measured by MTT assay. F) The apoptotic rate of SKMEL-28 and WM35 cells was measured by flow cytometry. G, H) The protein levels of Bcl-2, Bax, caspase 3, and cleaved-caspase 3 in SKMEL-28 and WM35 cells were detected by western blot. ${ }^{\star} \mathrm{p}<0.05$

in the expression levels of miR-33a-5p and SNAI2 in the resected tumor tissues by qRT-PCR and western blot. The results indicated that miR-33a-5p expression was upregulated (Figure $7 \mathrm{C}$ ), and the mRNA and protein expression levels of SNAI2 were markedly declined (Figures 7D, $7 \mathrm{E}$ ), suggesting that miR-33a-5p acted as an inhibitor of melanoma by regulating SNAI2 in vivo.

miR-33a-5p inhibited the PI3K/AKT/mTOR signaling pathway by regulation of SNAI 2 in melanoma. To investigate the role of the PI3K/AKT/mTOR signaling pathway and miR-33a-5p in melanoma, western blot was performed to detect the protein expression levels of key members of the $\mathrm{PI} 3 \mathrm{~K} / \mathrm{AKT} / \mathrm{mTOR}$ signaling pathway. The results demonstrated that the overexpression of miR-33a-5p remarkably inhibited the levels of p-PI3K, p-AKT308, and mTOR in
SKMEL-28 and WM35 cells compared with the controls, and the inhibitory effect of miR-33a-5p recovered by SNAI2 overexpression (Figures $8 \mathrm{~A}, 8 \mathrm{~B}$ ). Therefore, the data verified that miR-33a-5p negatively regulated the PI3K/AKT/mTOR signaling pathway by targeting SNAI2 in melanoma.

\section{Discussion}

Accumulating data have indicated that miRNAs have pivotal impacts on the occurrence and development of melanoma [23]. Many studies have shown that miRNAs could be used as markers for the diagnosis and treatment of melanoma [24]. Hence, it is necessary to study the molecular mechanism of how miRNAs modulated the progress 
A

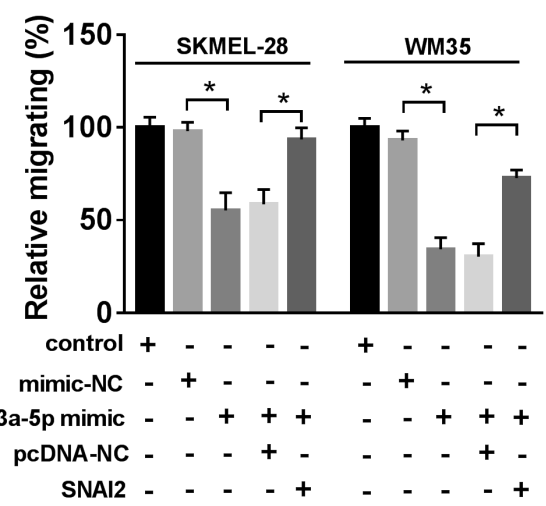

C
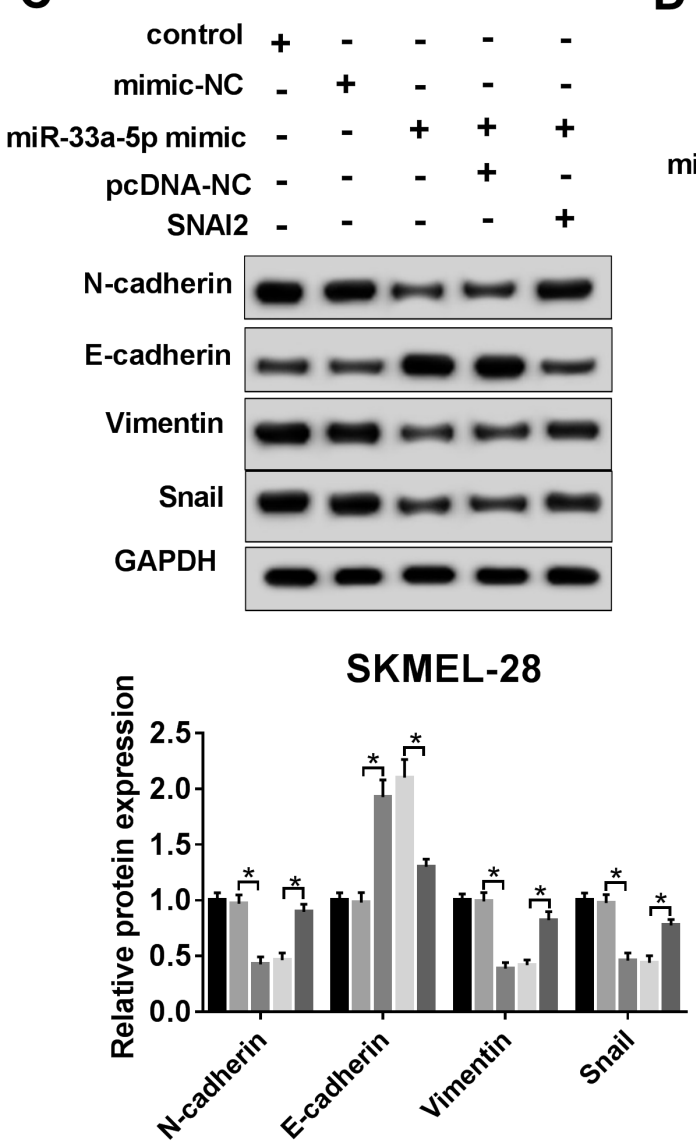

B

D
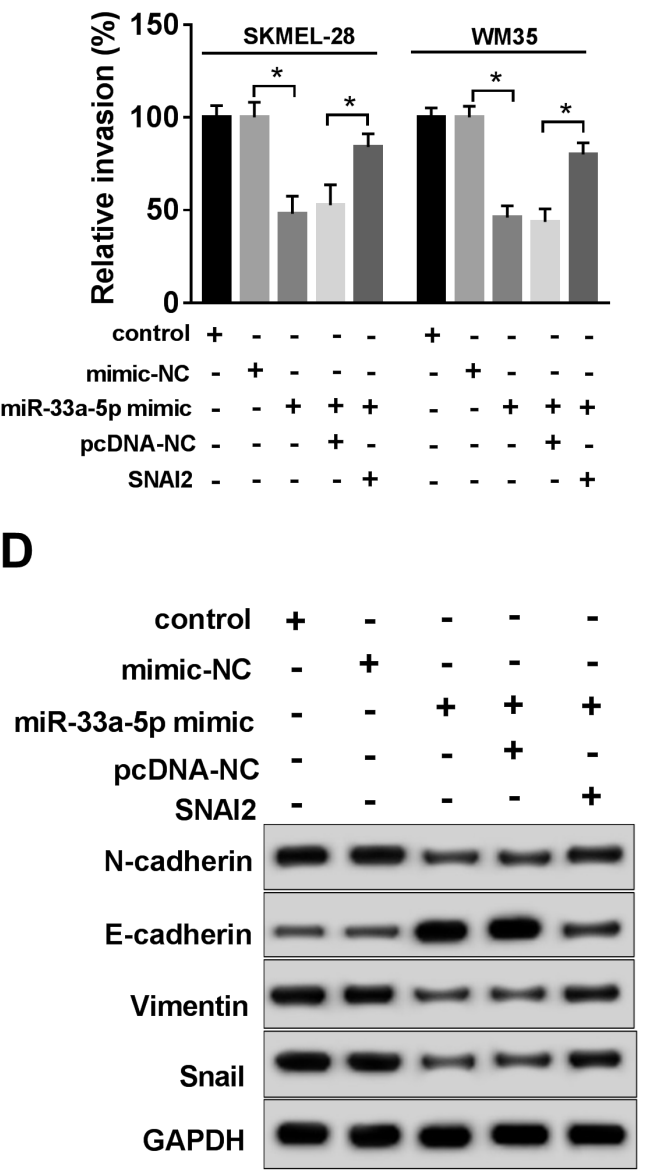

WM35

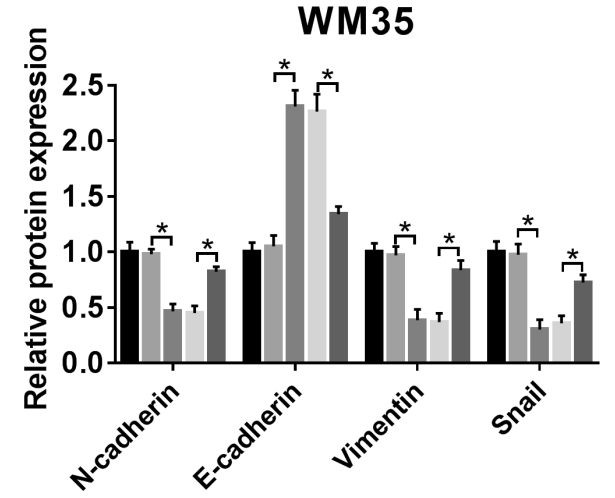

Figure 6. Overexpression of SNAI2 could regain the migration, invasion, and EMT repressed by miR-33a-5p. A, B) The migration and invasion of SKMEL-28 and WM35 cells transfected with control, mimic NC, miR-33a-5p mimic + pcDNA-NC or miR-33a-5p mimic + SNAI2 were measured by Transwell assay. C, D) The protein expression levels of $\mathrm{N}$-cadherin, E-cadherin, Vimentin, and Snail in SKMEL-28 and WM35 cells were measured by western blot. ${ }^{*} \mathrm{p}<0.05$

of melanoma. Our data indicated that miR-33a-5p was notably reduced in melanoma. Besides, miR-33a-5p mimic has retarded the growth, migration, invasion, and EMT of melanoma cells by targeting SNAI2. miR-33a-5p could also inhibit the PI3K/AKT/mTOR signaling pathway by regulating SNAI2. Our results identified that miR-33a-5p could serve as a novel key regulator of melanoma progression. 
A

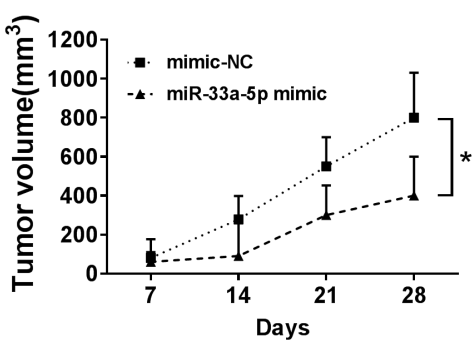

D

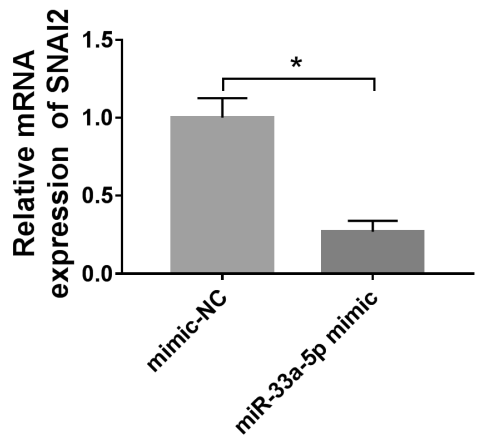

B

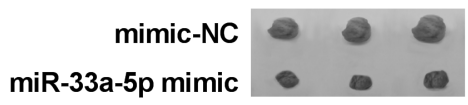

C

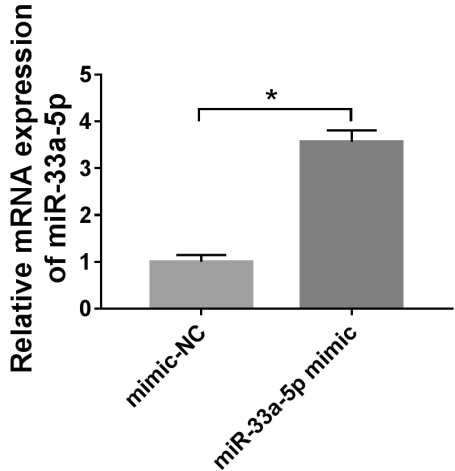

E
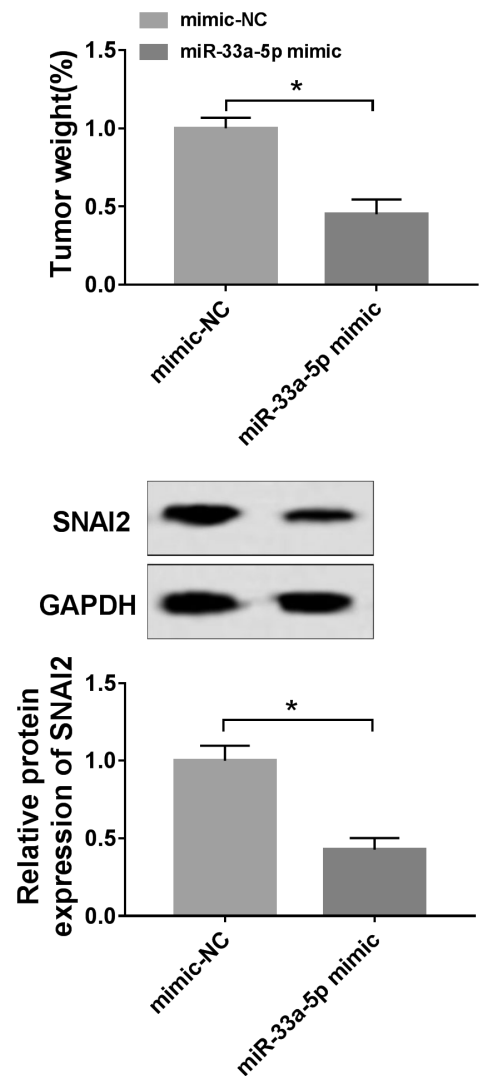

Figure 7. miR-33a-5p could impair tumor growth in vivo. A) Tumor volume was measured at the indicated time points after injection of SKMEL-28 cells transfected miR-33a-5p mimic or mimic NC. B) Tumor weight was measured at the endpoint of the xenograft experiments, and images of tumors from nude mice at autopsy are presented. C, D) The expression levels of miR-33a-5p and SNAI 2 were measured in the resected tumor tissues by $q$ RTPCR. E) The protein level of SNAI2 was measured in the resected tumor tissues by western blot. * $\mathrm{p}<0.05$

In previous studies, many miRNAs have been shown to participate in regulating the occurrence and development of melanoma. For example, miR-429 impeded melanoma cell proliferation and migration by targeting AKT1 [25]. miR-203 reinforced stemness properties in melanoma and enhanced tumorigenesis in vivo [26]. In our study, the results manifested that miR-33a-5p could regulate the progression of melanoma. The effect of miR-33a-5p in other types of tumors has been diffusely researched. It has been authenticated as a tumor-inhibiting factor in lung cancer [27], hepatocellular carcinoma [28], and osteosarcoma [29]. More than that, miR-33a-5p could hinder melanoma cell proliferation and elevated radiosensitivity by reversely modulating glycolysis in melanoma [15]. However, the impact of miR-33a-5p on melanoma progression was not clear enough. Our study showed that miR-33a-5p overexpression strikingly impaired growth, migration, invasion, and EMT. Addition- ally, in vivo experiments supported that miR-33a-5p could result in reduced tumor volume and weight, implying that miR-33a-5p repressed the progression of melanoma.

SNAI2 was a member of the SNAIL family of EMT associated transcription factors and accepted as an oncogene. Abnormally high expression of SNAI2 has been found in various cancers, including breast cancer [30], colorectal cancer [31], and melanoma [32]. Interestingly, Zhao et al. first discovered that SNAI2 could act as a tumor suppressor by repressing multidrug resistance via decreasing $A B C$ transporter genes in hepatocellular carcinoma cells [33]. In our study, we found that SNAI2 served as an oncogene and directly targeted by miR-33a-5p in melanoma, and miR-33a-5p could negatively modulate the expression of SNAI2. Moreover, the overexpression of SNAI2 recovered growth, metastasis, and EMT of melanoma cells suppressed by miR-33a-5p. Tian et al. indicated that SNAI2 regained 

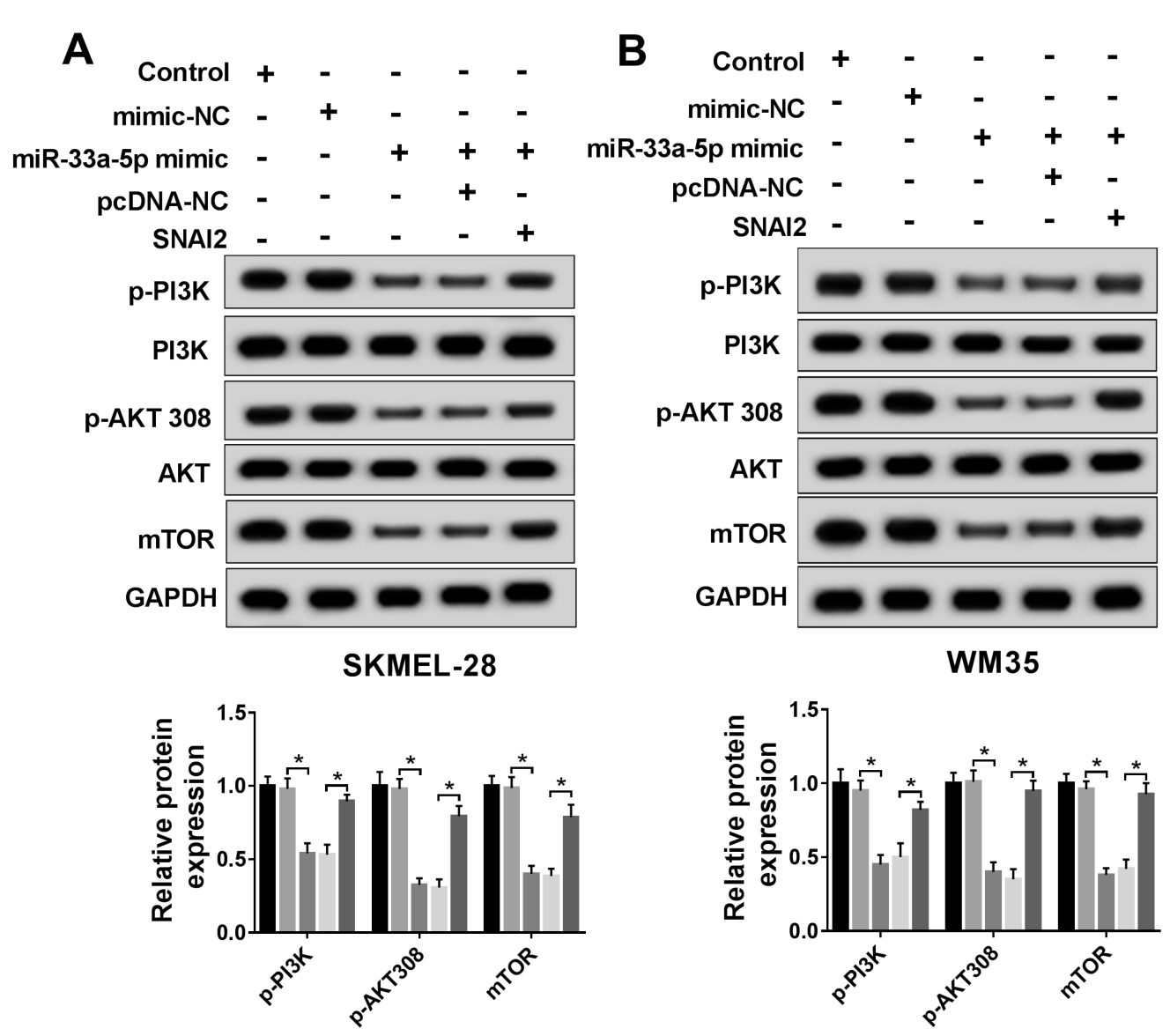

Figure 8. miR-33a-5p inhibited the PI3K/AKT/mTOR signaling pathway by regulating SNAI2 in melanoma. A, B) The protein expression levels of PI3K, p-AKT308, AKT, and mTOR were assessed by western blot in SKMEL-28 and WM35 cells transfected with control, mimic NC, miR-33a-5p mimic + pcDNA-NC or miR-33a-5p mimic + SNAI2. ${ }^{*} \mathrm{p}<0.05$

the inhibitory impacts of miR-203 on the growth, metastasis, and angiogenesis of prostate cancer cells [34], which was consistent with our findings. What's more, the PI3K/ AKT/mTOR signaling pathway has important roles in apoptosis, metastasis, and tumor genesis [35, 36]. And our results manifested that the miR-33a-5p overexpression inhibited the PI3K/AKT/mTOR signaling pathway via targeting SNAI2 in vitro.

To sum up, our results deemed that miR-33a-5p, as a tumor-inhibiting factor, suppressed the growth, metastasis, EMT and PI3K/AKT/mTOR signaling pathway activation of melanoma by targeting SNAI2, providing a new perspective for the diagnosis and treatment of human melanoma.

\section{References}

[1] SIEGEL R, MA J, ZOU Z, JEMAL A. Cancer statistics, 2014. CA Cancer J Clin 2014; 64: 9-29. https://doi.org/10.3322/ caac. 21208
[2] TROTTER SC, SROA N, WINKELMANN RR, OLENCKI T, BECHTEL M. A Global Review of Melanoma Follow-up Guidelines. J Clin Aesthet Dermatol 2013; 6: 18-26.

[3] MADDODI N, SETALURI V. Role of UV in cutaneous melanoma. Photochem Photobiol 2008; 84: 528-536. https://doi. org/10.1111/j.1751-1097.2007.00283.x

[4] PESSINA F, NAVARRIA P, TOMATIS S, COZZI L, FRANZESE $C$ et al. Outcome evaluation of patients with limited brain metastasis from malignant melanoma, treated with surgery, radiation therapy and targeted therapy. World Neurosurg 2017; 105: 184-190. https://doi.org/10.1016/j. wneu.2017.05.131

[5] GUPTA R, ARKATKAR T, KECK J, KOUNDINYA GK, CASTILLO $\mathrm{K}$ et al. Antigen specific immune response in Chlamydia muridarum genital infection is dependent on murine microRNAs-155 and -182. Oncotarget 2016; 7: 64726-64742. https://doi.org/10.18632/oncotarget.11461

[6] JULIA W, STEPHANIE J, SARINA K, GREGORY RI, SVEN D. Many roads to maturity: microRNA biogenesis pathways and their regulation. Nat Cell Biol 2009; 11: 228-234. https:// doi.org/10.1038/ncb0309-228 
[7] YANG T, THAKUR A, CHEN T, YANG L, LEI G et al. MicroRNA-15a induces cell apoptosis and inhibits metastasis by targeting BCL2L2 in non-small cell lung cancer. Tumour Biol 2015; 36: 4357-4365. https://doi.org/10.1007/s13277015-3075-1

[8] YAN K, GAO J, YANG T, MA Q, QIU X et al. MicroRNA34a inhibits the proliferation and metastasis of osteosarcoma cells both in vitro and in vivo. PLoS One 2012; 7: e33778. https://doi.org/10.1371/journal.pone.0033778

[9] YANG X, ZHAO H, YANG J, MA Y, LIU Z et al. MiR-150$5 \mathrm{p}$ regulates melanoma proliferation, invasion and metastasis via SIX1-mediated Warburg Effect. Biochem Biophys Res Commun 2019; 515: 85-91. https://doi.org/10.1016/j. bbrc.2019.05.111

[10] SUN Y, LI X, WANG H, WU J. MiR-431 is a prognostic marker and suppresses cell growth, migration and invasion by targeting NOTCH2 in melanoma. Eur Rev Med Pharmacol Sci 2019; 23: 3876-3884. https://doi.org/10.26355/eurrev_201905_17815

[11] MA M, DAI J, TANG H, XU T, YU S et al. MicroRNA-23a$3 p$ inhibits mucosal melanoma growth and progression through targeting adenylate cyclase 1 and attenuating cAMP and MAPK pathways. Theranostics 2019; 9: 945-960. https:// doi.org/10.7150/thno.30516

[12] YAN Y, ZHANG D, LEI T, ZHAO CA, HAN J et al. Micro RNA-33a-5p suppresses colorectal cancer cell growth by inhibiting MTHFD 2. Clin Exp Pharmacol Physiol 2019; 46: 928-936. https://doi.org/10.1111/1440-1681

[13] YANG J, SUN Y, GAO L, MENG Q, YANG B. Long non-coding RNA DANCR facilitates glioma malignancy by sponging miR-33a-5p. Neoplasma 2018; 65: 790-798. https://doi. org/10.4149/neo_2018_170724N498

[14] ZUO Z, MA L, GONG Z, XUE L, WANG Q. Long non-coding RNA CASC15 promotes tongue squamous carcinoma progression through targeting miR-33a-5p. Environ Sci Pollut Res Int 2018; 25: 22205-22212. https://doi.org/10.1007/ s11356-018-2300-z

[15] CAO K, LI J, CHEN J, QIAN L, WANG A et al. microRNA$33 a-5 p$ increases radiosensitivity by inhibiting glycolysis in melanoma. Oncotarget 2017; 8: 83660-83672. https://doi. org/10.18632/oncotarget.19014

[16] BARRALLO-GIMENO A, NIETO MA. The Snail genes as inducers of cell movement and survival: implications in development and cancer. Development. 2005; 132(14): 315161. https://doi.org/10.1242/dev.01907

[17] NIETO MA, SARGENT MG, WILKINSON DG, COOKE J. Control of cell behavior during vertebrate development by Slug, a zinc finger gene. Science 1994; 264: 835-839. https:// doi.org/10.1126/science. 7513443

[18] LIU BW, YU ZH, CHEN AX, CHI JR, GE J et al. Estrogen receptor- $\alpha$-miR-1271-SNAI2 feedback loop regulates transforming growth factor- $\beta$-induced breast cancer progression. J Exp Clin Cancer Res 2019; 38: 109. https://doi.org/10.1186/ s13046-019-1112-4

[19] SONG H, LIU L, SONG Z, REN Y, LI C et al. P4HA3 is epigenetically activated by slug in gastric cancer and its deregulation is associated with enhanced metastasis and poor survival. Technol Cancer Res Treat 2018; 17: 1533033818796485. https://doi.org/10.1177/1533033818796485
[20] GUPTA PB, CHARLOTTE K, JEAN-PHILIPPE B, SRIDHAR R, WEN-LIN $\mathrm{K}$ et al. The melanocyte differentiation program predisposes to metastasis after neoplastic transformation. Nat Genet 2005; 37: 1047-1054. https://doi. org/10.1038/ng1634

[21] ZHENG S, ZHENG L, LIU X, XING W, LIU X. Sinomenine inhibits the growth of melanoma by enhancement of autophagy via PI3K/AKT/mTOR inhibition. Drug Des Devel Ther 2018; 12: 2413-2421. https://doi.org/10.2147/DDDT. S155798

[22] LIM H, BAEK S, JUNG H. Bee venom and its peptide component melittin suppress growth and migration of melanoma cells via inhibition of PI3K/AKT/mTOR and MAPK Pathways. Molecules 2019; 24. pii: E929. https://doi.org/10.3390/ molecules24050929

[23] SITA-LUMSDEN A, DART DA, WAXMAN J, BEVAN CL. Circulating microRNAs as potential new biomarkers for prostate cancer. Br J Cancer. 2013; 108(10): 1925-30. https:// doi.org/10.1038/bjc.2013.192

[24] VARAMO C, OCCELLI M, VIVENZA D, MERLANO M, NIGRO CL. MicroRNAs role as potential biomarkers and key regulators in melanoma. Genes Chromosomes Cancer 2017; 56: 3-10. https://doi.org/10.1002/gcc.22402

[25] HUANG D, WANG F, WU W, LIAN C, LIU E. MicroRNA-429 inhibits cancer cell proliferation and migration by targeting the AKT1 in melanoma. Cancer Biomark 2019; 26: 63-68. https://doi.org/10.3233/CBM-190289

[26] SAHRANAVARDFARD P, FIROUZI J, AZIMI M, KHOSRAVANI P, HEYDARI R et al. MicroRNA-203 reinforces stemness properties in melanoma and augments tumorigenesis in vivo. J Cell Physiol 2019; 234: 20193-20205. https:// doi.org/10.1002/jcp.28619

[27] PAN J, ZHOU C, ZHAO X, HE J, TIAN H et al. A two-miRNA signature (miR-33a-5p and miR-128-3p) in whole blood as potential biomarker for early diagnosis of lung cancer. Sci Rep 2018; 8: 16699. https://doi.org/10.1038/s41598-01835139-3

[28] LI Y, CHEN G, YAN Y, FAN Q. CASC15 promotes epithelial to mesenchymal transition and facilitates malignancy of hepatocellular carcinoma cells by increasing TWIST1 gene expression via miR-33a-5p sponging. Eur J Pharmacol 2019; 860: 172589. https://doi.org/10.1016/j. ejphar.2019.172589

[29] JIANG N, WANG X, XIE X, LIAO Y, LIU N et al. lncRNA DANCR promotes tumor progression and cancer stemness features in osteosarcoma by upregulating AXL via miR-33a5p inhibition. Cancer Lett 2017; 405: 46-55. https://doi. org/10.1016/j.canlet.2017.06.009

[30] DU S, LI H, SUN X, LI D, YANG Y et al. MicroRNA-124 inhibits cell proliferation and migration by regulating SNAI2 in breast cancer. Oncol Rep 2016; 36: 3259-3266. https://doi. org/10.3892/or.2016.5163

[31] YANG P, CHEN T, XU Z, ZHU H, WANG J et al. Long noncoding RNA GAPLINC promotes invasion in colorectal cancer by targeting SNAI2 through binding with PSF and NONO. Oncotarget 2016; 7: 42183-42194. https://doi. org/10.18632/oncotarget.9741 
[32] VAND-RAJABPOUR F, SADEGHIPOUR N, SAEE-RAD S, FATHI H, NOORMOHAMMADPOUR P et al. Differential BMI1, TWIST1 , SNAI2 mRNA expression pattern correlation with malignancy type in a spectrum of common cutaneous malignancies: basal cell carcinoma, squamous cell carcinoma, and melanoma. Clin Transl Oncol 2017; 19: 489-497. https://doi.org/10.1007/s12094-016-1555-4

[33] ZHAO XY, LI L, WANG XB, FU RJ, LV YP et al. Inhibition of snail family transcriptional repressor 2 (SNAI2) enhances multidrug resistance of hepatocellular carcinoma cells. PLoS One 2016; 11: e0164752. https://doi.org/10.1371/journal. pone. 0164752
[34] TIAN X, TAO F, ZHANG B, DONG JT, ZHANG Z. The miR-203/SNAI2 axis regulates prostate tumor growth, migration, angiogenesis and stemness potentially by modulating GSK-3 $\beta / \beta$-CATENIN signal pathway. IUBMB Life 2018; 70: 224-236. https://doi.org/10.1002/iub.1720

[35] TANG H, LI RP, LIANG P, ZHOU YL, WANG GW. miR125a inhibits the migration and invasion of liver cancer cells via suppression of the PI3K/AKT/mTOR signaling pathway. Oncol Lett 2015; 10: 681-686. https://doi.org/10.3892/ ol.2015.3264

[36] ZHU P, LIAO LY, ZHAO TT, MO XM, CHEN GG et al. GPER/ERK\&AKT/NF- $\kappa$ B pathway is involved in cadmiuminduced proliferation, invasion and migration of GPERpositive thyroid cancer cells. Mol Cell Endocrinol 2017; 442: 68-80. https://doi.org/10.1016/j.mce.2016.12.007 